\title{
Application of media literacy education for changing attitudes about self-medication of slimming supplements
}

\author{
Mahnaz Solhi ${ }^{1}$, Hanieh Jormand ${ }^{2 *}$, Mahmood Reza Gohari ${ }^{3}$ \\ Received: 12 Mar 2016 \\ Published: 22 Dec 2017
}

\begin{abstract}
Background: Self-medication of slimming supplements has increased, and media advertising has a role in this. The present study aimed at determining the effect of media literacy educational intervention on attitudes about self-medication of slimming supplements among the female students at dormitories of Iran University of Medical Sciences.

Methods: In this quasi-experimental study with a control group, 98 students were selected by multistage cluster sampling method and were randomly divided into 2 equal groups of test and control. The data collecting instrument was a researcher-made questionnaire about media literacy and attitude on self-medication of slimming pills. Based on the results obtained from the early completion of the questionnaires, an appropriate educational intervention was designed and implemented. Data were analyzed 1 and 3 months after the intervention using the following statistical methods: repeated measurements, Friedman test, Chi square, independent T-test and MannWhitney test.

Results: Before the intervention, the mean scores of attitude and dimensions of media literacy were moderate in both groups, and there was no significant difference between them. However, positive correlations were observed among attitude and knowledge $(\mathrm{r}=0.99, \mathrm{p}=0.001)$, critical thinking $(\mathrm{r}=0.61, \mathrm{p}=0.001)$, and analysis $(\mathrm{r}=0.37, \mathrm{p}=0.009)$ in the experimental group. The mean scores of attitudes and dimensions of media literacy were found to be significant in both groups 1 and 3 months after the intervention $(\mathrm{p}<0.05)$.
\end{abstract}

Conclusion: Media literacy education was effective in promoting a positive attitude about slimming supplements.

Keywords: Media literacy, Attitude, Slimming supplements, self-medication, Female student

Copyright $\odot$ Iran University of Medical Sciences

Cite this article as: Solhi M, Jormand H, Gohari MR. Application of media literacy education for changing attitudes about self-medication of slimming supplements. Med J Islam Repub Iran. 2017 (22 Dec);31:119. https://doi.org/10.14196/mjiri.31.119

\section{Introduction}

Nowadays, the use of slimming supplements as a treatment for obesity is common (1). These supplements have various forms (tablets, powders, liquids, capsules, and other forms) and are sold in the market. In a study on health beliefs in nutritional supplements, $80.3 \%$ of the participants had taken slimming supplements in the past 2 years, and $28.8 \%$ were suffering from severe complications of taking these pills. In the present study, a positive correlation was found between attitudes in controlling and consuming slimming supplements (2). In another study, illegal herbal slimming compounds were found in Chinese

\section{Corresponding author: Hanieh Jormand,jormand69h@gmail.com}

1. Health Promotion Research Center, Iran University of Medical Sciences, Tehran, Iran.

2. School of Health, Iran University of Medical Sciences, Tehran, Iran.

3. Department of Biostatistics, School of Health, Iran University of Medical Sciences, Tehran, Iran. herbal capsules and pills for weight loss. Illegal herbal slimming compounds were also found in herbal slimming pills. These so-called herbal medicines, which are commonly advertised on satellites channels, have been made from unauthorized substances and cannot be trusted based on their packaging. The side effects of these compounds have been reported as dry mouth, tremors, increased blood pressure, sleep disorders, loss of appetite, heartbeat, liver and kidney damage, cardiovascular problems, and tendency to reuse, and even death (3). Informing consumers about the proper use of medicines and preventing self-

$\uparrow$ What is "already known" in this topic:

Slimming supplements have many side effects. Education in the field of slimming supplements consumption has been emphasized because these products do not have the desired outcome. Providing useful information to consumers about the proper use of medicines and preventing self-medication are of paramount importance.

$\rightarrow$ What this article adds:

Media literacy training is effective in improving the attitude and aspects of media literacy about slimming supplements and self-medication. 
medication are of paramount importance (4).

In various studies, providing education in the field of slimming supplements consumption has been emphasized because these products do not have the desired outcome. They do not contain natural compounds and are supplied in inappropriate, but accessible places, such as supermarkets and stores (5). Advertising plays an important role in taking supplements and diet control (3). There are several reasons for consuming slimming supplements in Iran including urbanization, sedentary lifestyle as a result of industrial life, and the aging population, which lead to consumption of supplements, especially slimming supplements (6).

Media literacy is the ability to access, analyze, evaluate, and communicate the media information and messages that can be provided in various printed and no printed frameworks. Media literacy is one of the most important phenomena of the information age that helps the audience enhance their level of knowledge and use media, not only out of habit and recreation, but also for use and interpretation of the media messages with insight (7). Three aspects of media literacy include awareness promotion of the content of media, second are critical watching instruction, and the social, economic, and political analysis of the media that are not visible at first glance.

The first point about the issue of media literacy is to pay attention to consumption level and ratio of each media in the media rainbow; second point is to see, read, and hear mainly through a critical perspective; and the third point is the role of stakeholders and owners of the media (8). In Canadian educational system, media literacy has been accepted officially for better understanding and perception and for critical freedom of action. In Japan, media literacy education was implemented by the Ministry of Post and Telecommunications for the first time, taking the responsibility to provide and promote media literacy education. Media literacy education in these 2 countries has led their students to gain greater understanding of the media outlets, compared to students in other countries. They are well aware what they want from the media, and therefore, are actively engaged in critically analyzing and evaluating the media content to understand the content of the messages encountered (9). In a study by Brian et al. an independent association was found between media literacy and reductions in smoking among adolescents and reduced susceptibility to smoke in future (10). In a review by Brown et al. in 2006, media literacy was counted as the potential for improving health among adolescents (11). In a study by Zarepour et al., the role of mass media in promoting health literacy in the society was mentioned (12). Media literacy is considered as an important tool in the direction of human development and the sustainable development of the communities (13). Low levels of media literacy in one community are associated with a prevalence of many health problems including communicable and non-communicable diseases.

These days, media literacy has been evaluated by experts as an important indicator to determine the general status of public health. Health education with the aim of raising the level of media literacy empowers people to decide about their own health, family, and community and adopt healthy behaviors, improving public health. Female students are at risk of taking slimming supplements, and providing education for them is essential to prevent the use of these supplements in this group. This study aimed at determining the effect of media literacy on the female students' attitude about self-medication of slimming supplements in the dormitories of Iran University of Medical Sciences. Studies at the researcher's organizational association was the reason behind conducting the study in this university. The results of this study can be applied in designing interventions to enhance students' knowledge and attitudes, and thus prevent slimming supplements selfmedication.

\section{Methods}

A total of 98 female students staying at dormitories of Iran University of Medical Sciences participated in this interventional controlled study.

To calculate the sample size in this study, the formula for comparison of means in 2 independent groups was used. In this formula, the standard deviation of both control and the experimental groups was considered equal, and the sample size was estimated by leaving the mean difference of attitude, awareness, body image, and selfesteem, considering the largest obtained value of the formula and excessive sample to prevent the sample loss. To that end, standard deviation was taken from the previous study as 17 (14), and the mean difference (attitude) was considered to be10; the minimum sample size required in each test and control group was calculated to be 45; however, taking into account the excessive $10 \%$ to avoid sample loss, the sample number in each group was estimated to be 49 , providing a total of 98 .

Sampling was random, that is, a list of medical sciences dormitories was provided (4 dorms), then, 2 were randomly selected and divided into experimental and control. In each dorm, 49 students were enrolled randomly in the study. Data collection instruments in the present study were 4 questionnaires:

1. The author designed attitude questionnaire on selfmedication of slimming pills; it contains 12 questions on attitudes about prevention of self-medication of slimming supplements, rated on a 5- point Likert scale, (strongly agree, agree, no comment, disagree, and strongly disagree); the minimum and maximum scores were 12 and 60 , respectively. Content validity test was used to validate the questionnaire through a panel session with 10 experts, and the questions were altered based on the results. Also, face validity was determined based on the experts' comments. Content validity index (CVI) acquired from all the questions in this section was higher than 0.75 , and content validity ratio (CVR) obtained from all the questions in this section was 0.62 , which was appropriate in accordance with Lawshe table (for 10). To determine the reliability of the attitudes questionnaires, we asked 10 participants, out of the sample, to complete the questionnaire twice within 10 days; the obtained coefficients for Cronbach's Alpha test for questions was 0.78 , and the correlation coefficient of the attitudes scores was 0.87 , based on test-retest. 
Benchmark category for attitudes scores was mean $\pm \mathrm{SD}$. Accordingly, the points less than 28.01 were weak, and points between 28.02 and 46.16 were neutral and those higher than 46.17 were considered desirable.

2. The author designed media literacy questionnaire in 3 parts as follow: media management awareness, critical thinking, and media analysis about media self-medication of slimming supplements. In designing this questionnaire, the results obtained by Haddad et al. in assessing the students' media literacy were considered. The questionnaire contains 14 multiple-choice questions on media management knowledge, the maximum score of which belonged to the most relevant questions. Minimum and maximum scores were 14 and 80 . Benchmark category for attitudes scores was mean \pm standard deviation. Accordingly, scores less than 35.45 were weak, scores between 35.46 and 49.76 medium, and higher than 49.76 were desirable. Critical thinking consists of 12 questions with 5 scales (very low, low, medium, high, and very high). The minimum and maximum points in this section were 12 and 60 , respectively. To categorize the scores, the mean \pm standard deviation was used; the scores less than 29.56 were considered weak, those between 29.57 and 43.41 medium, and higher than 43.51 were favorable. The analysis section consisted of 10 questions with 5 scales (very low, low, medium, high, and very high). The minimum and maximum points in this section were 10 and 50, respectively. Benchmark category for attitudes scores was mean \pm standard deviation. Thus, the scores less than 23.38 were weak, those between 23.39 and 36.47 medium, and higher than 57.36 were favorable. To assess the validity of media literacy questionnaire, content validity was used in a way that the questionnaire was given to 10 experts in health education, and changes were made based on their opinion. Validity index (CVI) was acquired from all the questions in this section and was higher than 0.75; and validity ratio (CVR) acquired from all the questions in this section was 0.62 , which was appropriate in accordance with the Lawshe table (for 10). Content validity test was used to validate the questionnaire by panel session with 10 experts, and the questions were changed based on the results. Also, face validity was based on the experts' comments. To determine the reliability of the media literacy questionnaires, they were completed by 10 students, out of the sample, twice within 10 days, and the obtained coeffi- cients for Cronbach's Alpha test for all questions was higher than 0.76 . Furthermore, the obtained coefficient on the test-retest of awareness and media perception was $\mathrm{r}=0.64$, in the critical thinking section it was $\mathrm{r}=0.64$, and it was $r=0.84$ in the analysis section (15).

Moreover, the demographic characteristics including age, marital status, educational level, field of study, and BMI (Body Mass Index= BMI) were obtained from the participants.

The questionnaires were completed by all the individuals in both groups at dorms. Based on the results analysis of the pretest, learning course of media literacy was designed and modified according to the experts' view. Then, two 45-minute training sessions were provided on the prevention of self-medication of slimming supplement that included a short lecture, Q \& A, group discussion, and nutrition advice by means of educational videos, blogs, and pamphlets only for the experimental group. To prevent any communication between experimental and control groups, the individuals of the 2 groups were selected from different dormitories.

Data were analyzed in (16) SPSS using descriptive statistics (frequency, percentage, mean, and standard deviation) and analytical tests (repeated measurements, Friedman test, Chi square, independent T-test and MannWhitney test).

To ensure ethical consideration, administrative correspondence and coordination were made with officials of the 2 dorms. Written informed consent was obtained from all the participants and they were assured about confidentiality of the data. Participation was voluntary, and the participants could leave the study at any time. They were instructed how to fill out the questionnaire. In this study, confounding variables were controlled by random allocation sampling method and balancing cases. Factors affecting the internal validity of the study, such as maturity date and time, were controlled by a control group and shortening research time, moreover, factors affecting the external validity of the study were controlled by an increase in the interval between tests.

\section{Results}

Mean and standard deviation of age in the experimental and control groups was $4.84 \pm 24.71$ and $2.66 \pm 27.04$, respectively. Moreover, the mean and standard deviation of

\begin{tabular}{|c|c|c|c|c|c|c|}
\hline \multirow[t]{2}{*}{ Variable } & \multirow[t]{2}{*}{ Category } & \multicolumn{2}{|c|}{ Case } & \multicolumn{2}{|c|}{ Control } & \multirow{2}{*}{$\begin{array}{c}\text { Chi square test } \\
\text { p.value }\end{array}$} \\
\hline & & Number & Percent & Number & Percent & \\
\hline \multirow[t]{3}{*}{ Age } & Younger than 20 & 5 & 10.2 & 0 & 0 & 0.06 \\
\hline & $20-30$ & 38 & 77.6 & 39 & 79.9 & \\
\hline & Older than 30 & 6 & 12.22 & 10 & 20.4 & \\
\hline \multirow[t]{3}{*}{ BMI } & Under 20 slim & 8 & 16.3 & 5 & 10.2 & 0.074 \\
\hline & 20-30 medium & 24 & 29 & 35 & 71.4 & \\
\hline & over 25 fat & 17 & 34.7 & 9 & 18.4 & \\
\hline \multirow[t]{2}{*}{ Marital status } & Single & 41 & 83.7 & 36 & 73.3 & 0.16 \\
\hline & Married & 8 & 16.3 & 13 & 26.5 & \\
\hline \multirow[t]{2}{*}{ Education } & BA & 27 & 55.1 & 19 & 38.8 & 0.08 \\
\hline & MA \& PhD & 2 & 44.9 & 30 & 61.2 & \\
\hline \multirow[t]{2}{*}{ Field of study } & Health & 45 & 91.8 & 23 & 46.9 & 0.001 \\
\hline & Paramedical and Medical & 4 & 8.2 & 26 & 53.1 & \\
\hline
\end{tabular}


BMI in the experimental and control groups was $3.11 \pm 23.03$ and $2.65 \pm 23.16$, respectively. Other demographic characteristics of the studied individuals are demonstrated in Table 1.

Participants were selected from 2 different dorms to prevent communication between the experimental and control groups; this resulted in a difference in the field of the study in the 2 groups, and ultimately significant comparison was justified in terms of that distinction. Using Kolmogorov- Smirnov test, it was determined that the variables of marital status, education, and self-esteem were not normally distributed. Using Pearson correlation coefficient before the intervention in the experimental group, we found that there was a positive correlation among attitude and knowledge $(\mathrm{r}=0.99, \mathrm{p}=0.001)$, critical thinking $(r=0.61, p=0.001)$, and analysis $(r=0.37$, $\mathrm{p}=0.009$ ). That is, the students who scored well in attitude also scored well in critical thinking and analysis. Based on Spearman test, before the intervention, a significant positive correlation was observed between knowledge and educational level $(p=0.004, r=0.4)$, indicating that the students with higher education level had higher awareness scores. Also, according to the results of Chi square test, no significant correlation was found among the variables of attitude, body image, awareness, critical thinking, and analysis with demographic variables. Before the intervention, the mean of the variables of attitude, body image, self-esteem, and dimensions of media literacy in both groups was moderate.

Comparison of the mean scores of attitude, media management awareness, study skills, critical thinking, and media analysis before and 1 month after the intervention revealed a significant difference between the mean scores of attitude, and dimensions of media literacy 1 month after the intervention (Table 2).

Comparison of the mean scores of attitude, media management knowledge, critical thinking, and media analysis before and 3 months after the intervention showed significant differences (Table 3).

Table 2. The mean and standard deviation of the main variables evaluated before and 1 month after intervention in the experimental and control groups, IUMS, 2015

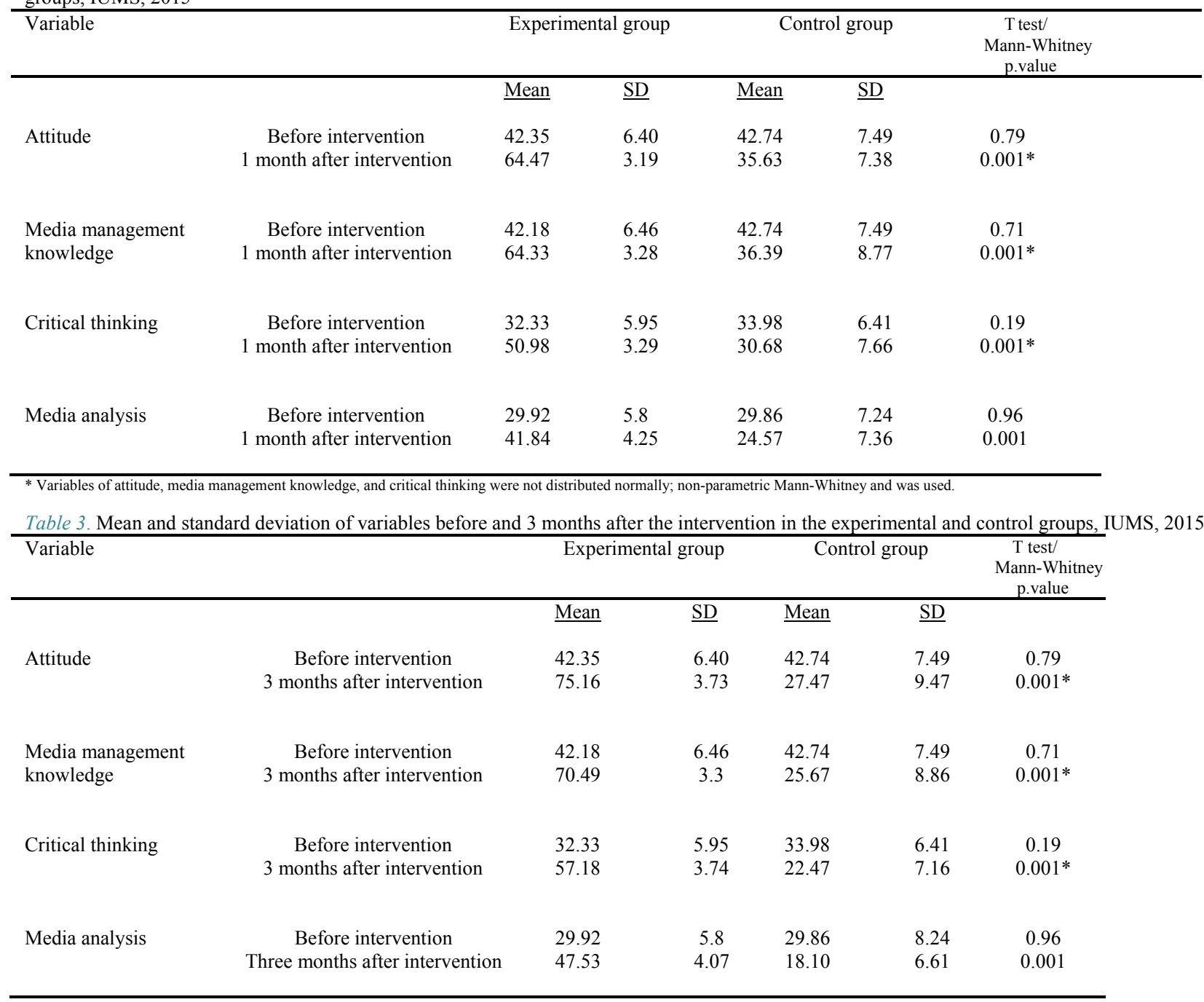

* Variables of attitude, media management knowledge, and critical thinking were not distributed normally; non-parametric Mann-Whitney was used. 
Table 4. Mean and standard deviation of various dimensions of media literacy 1 and 3 months after the intervention in the experimental and control groups, IUMS, 2015

\begin{tabular}{|c|c|c|c|c|c|c|c|c|}
\hline \multirow[t]{2}{*}{ Variable } & & \multicolumn{3}{|c|}{ Experimental group } & \multicolumn{2}{|c|}{ Control group } & \multicolumn{2}{|c|}{$\begin{array}{c}\text { T-test/ } \\
\text { Mann-Whitne } \\
\text { p.value }\end{array}$} \\
\hline & & Mean & Category & $\underline{\mathrm{SD}}$ & $\underline{\text { Mean }}$ & Category & $\underline{\mathrm{SD}}$ & \\
\hline \multirow[t]{2}{*}{ Attitude } & 1 month after intervention Category & $\overline{64.37}$ & Good & $\overline{3.19}$ & $\overline{35.63}$ & Middle & $\overline{7.38}$ & $0.001 *$ \\
\hline & 3 months after intervention Category & 75.16 & Good & 375 & 27.47 & Poor & 9.47 & $0.001 *$ \\
\hline \multirow{2}{*}{$\begin{array}{l}\text { Media management } \\
\text { knowledge }\end{array}$} & 1 month after intervention Category & 64.33 & Good & 6.39 & 36.39 & Middle & 8.77 & $0.001 *$ \\
\hline & 3 months after intervention Category & 70.49 & Good & 3.3 & 25.67 & Poor & 8.86 & $0.001 *$ \\
\hline \multirow[t]{2}{*}{ Critical thinking } & 1 month after intervention Category & 50.98 & Good & 3.29 & 30.68 & Middle & 7.66 & $0.001 *$ \\
\hline & 3 months after intervention Category & 57.18 & Good & 3.74 & 22.47 & Poor & 7.16 & $0.001 *$ \\
\hline \multirow[t]{2}{*}{ Media analysis } & 1 month after intervention Category & 41.84 & Good & 4.25 & 24.57 & Middle & 7.36 & $0.001 *$ \\
\hline & 3 months after intervention Category & 47.53 & Good & 4.07 & 18.10 & Poor & 6.61 & $0.001 *$ \\
\hline
\end{tabular}

Based on repeated measurement test and its nonparametric counterpart test (Fredman test), a significant difference was found among the mean scores of attitude, media management knowledge, critical thinking, and media analysis in 3 phases, before the intervention, 1 month, and 3 months after intervention in the 2 groups $(p<0.001$, for all). The mean scores of attitude and media literacy dimensions reached a good level 1 month after the intervention. Moreover, the mean scores of all variables increased 3 months after the intervention.

Comparison between the mean scores of attitude, media management knowledge, critical thinking, and media analysis 1 and 3 months after the intervention showed significant differences (Table 4).

\section{Discussion}

The present study aimed at determining the effect of media literacy on the attitude of 98 female students staying at 2 dormitories of Iran University of Medical Sciences about self-medication of slimming supplements during 2014 and 2015. The average age of the studied students in the experimental and control groups was 24 and 27 years, respectively, and BMI indicator in the experimental and control groups was about 23. The percentage of single participants in the intervention group was 83.7, and it was 73.5 in control group. Of the participants in the intervention and control groups, $16.3 \%$ and $26.5 \%$ were married, respectively. Moreover, $8.2 \%$ of the participants in the intervention group and $53.1 \%$ in the control group were studying in the health field. Furthermore, 91.8 of the participants in the intervention group and 46.9 in the control group were studying in non-health fields. Of the participants, $55.1 \%$ in the intervention group and 38.8 in the control group were under-graduates; and $44.9 \%$ in the control group and 61.2 in the intervention group were post-graduates. Comparison of field variables between the 2 groups showed no significant difference in the 2 groups before the intervention. The 2 groups did not differ in attitude, media management knowledge, critical thinking, and media analysis. Before the intervention, the mean scores of attitude, media management knowledge, critical thinking, and analysis were at the medium level, and the 2 groups showed no statistically significant differences. However, 1 month after the intervention, the mean scores of these variables reached a good level, and a statistically significant difference was observed between the mean scores of the variables in the two groups. Also, 3 months after the training intervention, the scores of the variables in the test group were good and there was still a significant difference between the mean of these variables. In a study entitled, "teaching media literacy in children and adolescents" by Taherian, it was found that media could enable the citizens, especially children and adolescents, to develop critical thinking and creative thinking skills in a way that they began to produce the information (7).

In a study by Haddad et al. on the evaluation of students' media literacy in Tehran during 2011 and 2012, it was found that the studied participants were poor in dimensions of perception and knowledge, and they needed to be trained (15). The results obtained by Bakhtiarizadeh in a review of the information literacy of students in AL-Zahra University in 2002 indicated that information literacy of the research community was low, and there was a significant difference between academic disciplines of the studied community in information literacy (16). Overall, findings of the current study revealed that media literacy of the studied students was not good, and designing interventions in this area was required for these students.

In the present study, the executed educational intervention led to an increase in the mean scores of attitude, media management knowledge, critical thinking, and analysis 1 month and 3 months after the intervention. Many similar studies including the studies by Singer and Zuckerman in 1980, Kan Wemster in 1992, and Johnson in 1997, and Yatz Vastine in 2000 also showed the effectiveness of media literacy (7). Brian et al.'s study in 2006 on the relationship between smoking and the role of media literacy indicated the effective role of media literacy in cutting down on smoking (10). This was in line with the results of the study by Dehdari et al. on the effectiveness of media literacy in decreasing violence among the users of computer games.

Score of attitude in the intervention group increased 1 month and 3 months after the intervention, while in the control group no decrease was observed after the intervention (17). Findings of the current study implied the impact of the executed intervention on improving the attitudes of the participants in the control group, thus, designing intervention programs to enhance the students' attitudes is 
needed.

Scores of management knowledge, media, critical thinking, and analysis in experimental and control groups before the study were at a moderate level; however, in the assessment of media literacy of students of Isfahan University of Medical Sciences, the overall mean score of media literacy was $3.34 \pm 0.444$, which was above average and reported as good (18). Based on the results of the present study, designing a program to promote media literacy of students is of prime importance.

Comparison of the mean scores of variables of attitude, media management knowledge, critical thinking, and media analysis in 3 stages (before the intervention, 1 month, and 3 months after intervention), using the repeated measurements Friedman test and its non-parametric counterpart Friedman test, showed that the mean scores of media literacy dimensions reached a good levellmonth after the intervention. However, the mean scores for all variables increased 3 months after the intervention. Increasing the mean scores of these variables in both groups reflected the continuing impact of the training.

In the current study, there was a significant positive correlation among attitude and awareness, critical thinking, and analysis. Based on these findings, improvement of the students' media literacy can affect development of their attitude. In the present study, a positive correlation was observed between knowledge and educational level. Thus, for students, this awareness development about the prevention of taking slimming supplements is a priority at lower educational levels.

One of the main limitations of this study was that it was conducted on female students living in dormitories of Iran University of Medical Sciences. Thus, it is suggested that similar studies be conducted on students not living in dorms, studying in other disciplines, and on male students who take bodybuilding supplements. The self-reporting and quantitative nature of this study was another limitation of the current study. Conducting qualitative studies with interviews is also suggested for future studies.

\section{Conclusion}

Media literacy training has been effective in improving the status of the studied students' attitude and aspects of media literacy about self-medication of slimming supplements. When designing media literacy education interventions, students with lower education levels should be prioritized.

\section{Acknowledgment}

The present research project (No.25133) has been approved by the Vice Chancellor for Research and Technology in Iran University of Medical Sciences. Authors would like to acknowledge the financial support of the Vice Chancellor and cooperation of the female students at Iran University of Medical Sciences, who participated in the study.

\section{Conflict of Interests}

The authors declare that they have no competing interests.

\section{References}

1. Politer JL, Shiffman S, Rohay JM, Harkins AM, Burton SL, Wadden TA. Use of Dietary Supplements for Weight Loss in the United States: results of a national survey. Obesity. 2008; 16(4):790-796.

2. Sievers JA. Nutrition \&health beliefs in food supplement use theory a study of the health locus of control theory (vitamins, habits minerals, toxicity). A thesis submitted to the faculty of the department of nutrition \& food science. University of Arizona; April 1985. Persistent link: http://hdl.handle.net/10150/291271

3. Azizi F, Asgari S, Hedayati M, Khazan M. Evaluation of potentially harmful stimulant found in Chinese internal marketing herbal weightloss supplements. Ann inter MED SBUMS Re mag. 2012;35(4):209214. (Persian)

4. Nasiriasl M. A survey of medications without a prescription in the home. JQUMS. 2008;2(11):22-26.

5. Skiba-King EW. Vitamins, herbs, and supplements: tools of empowerment, J Psychosoc Nurs Ment Health Serv. 2001;9(4):34-41.

6. Karblayyzadeh AS. What price is excessive imports Food. Health News. 2013 [cite 2013 August 19]. Access in: http://tnews.ir/news/ D14916642272.html

7. Amiri R. Health literacy book research. Tehran: Strategic Research Institute; 2008. Access in: www.csr.ir. (Persian)

8. Shahbazi M. What is media literacy? Tehran: Ministry of Culture and Islamic Guidance; 2011. [2011/8/23] Access in: http://rasaneh. farhang.gov.ir/fa/newsagency/1636(Persian)

9. Nasiri B, Aghili V. Measuring in Acquisition of Media Literacy skills in japan and Canada. Q J Educ Innovat. 2012;41. (Persian)

10. Primack BA, Gold MA, Land SR, Fine MJ. Association of Cigarette Smoking and Media Literacy about Smoking among Adolescents. J of Adult Health. 2006;39(4):465-472.

11. Brown Jane D, Knight James L. Media Literacy Has Potential to Improve Adolescents' Health. J of Adolt Health. 2006;39(4):459-460.

12. Zareipour MA, Zareipur M, Movahed A. Role of Mass Communication in Improvement Media Literacy Society, Tehran: Strategic Research Institute; 2008. Access in: www.csr.ir. (Persian)

13. Babaii rad B. Media Literacy \& Human Development Improvement Research Bulletin. 2008;22. Access in: www.csr.ir. (Persian)

14. Khazir Z, Dehdari T. Attitude of female medical students towards cosmetic surgery and its relation with body image. Razi J Med Sci 2013;20(117):1-9. (Persian)

15. Haddad Z. Assessment of media literacy in students of high school and university in Tehran. J Res Pers. 2012;16:52-74. (Persian)

16. Bakhtiarzadeh A. Information literacy in Student of Alzahra University. [Dissertation].St. Vank: Alzahra University; 2002. (Persian)

17. Dehdari T, Shojaei $\mathrm{S}$. The effectiveness of media literacy in reducing violence in the subsidy game users. A thesis submitted to the faculty of the department of Education health \& promotion health. [Dissertation].St. Ghods: Tehran University of Medical science; 2012. (Persian)

18. Ashrafi Rezii H, Ramezani A, Aghajani H, Kazempour Z. A survey of media literacy \& information situation in medicine University students. Information systems and services quarterly. 2013;2(26). (Persian) 